\title{
Bayesian inference for the left truncated exponential distribution based on pooled Type-II censored samples
}

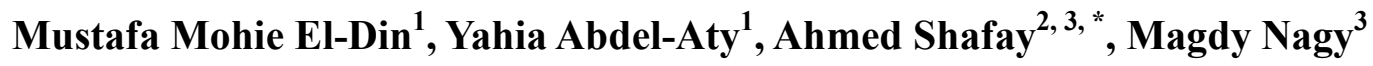 \\ ${ }^{1}$ Department of Mathematics, Faculty of Science, Al-Azhar University, Cairo, Egypt \\ ${ }^{2}$ Nature Science Department, Community College of Riyadh, King Saud University, P.O. Box 28095, Riyadh 11437, Saudi Arabia \\ ${ }^{3}$ Department of Mathematics, Faculty of Science, Fayoum University, Fayoum, Egypt
}

\section{Email address:}

mmmmoheeldin@yahoo.com (M. M. El-Din), yahia1970@yahoo.com (Y. Abdel-Aty), a_shafay2013@yahoo.com (A. Shafay), mn112@fayoum.edu.eg (M. Nagy)

\section{To cite this article:}

Mustafa Mohie El-Din, Yahia Abdel-Aty, Ahmed Shafay, Magdy Nagy. Bayesian Inference for the Left Truncated Exponential Distribution Based on Pooled Type-II Censored Samples. American Journal of Theoretical and Applied Statistics. Vol. 3, No. 6, 2014 , pp. $202-210$. doi: $10.11648 /$ j.ajtas.20140306.15

\begin{abstract}
In this paper, the maximum likelihood and Bayesian estimations are developed based on the pooled sample of two independent Type-II censored samples from the left truncated exponential distribution. The Bayesian estimation is discussed using different loss functions. The problem of predicting the failure times from a future sample from the sample population is also discussed from a Bayesian viewpoint. A Monte Carlo simulation study is conducted to compare the maximum likelihood estimator with the Bayesian estimators. Finally, an illustrative example is presented to demonstrate the different inference methods discussed here.
\end{abstract}

Keywords: Bayesian Estimation, Pooled Type-II Censored Samples, Left Truncated Exponential Distribution, Bayesian Prediction, Maximum Likelihood Estimation

\section{Introduction}

In reliability analysis, experiments are often terminated before all units on test fail based on cost and time considerations. In such cases, failure information is available only on part of the sample, and only partial information on all units that had not failed. Such data are called censored data. There are several forms of censored data. One of the most common forms of censoring is Type-II right censoring which can be described as follows: Consider $n$ identical units under observation in a life-testing experiment and suppose only the first $r \leq n$ failure times $X_{1: n}, X_{2: n}, \ldots, X_{r: n}$ are observed and the rest of the data are only known to be larger than $X_{r: n}$. In Type-II censoring scheme, if $r$ is small and $n$ is relatively large compared to $r$, the precision of the estimates of parameters obtained from such a censored data will be very low. In such a situation, if it will be possible and convenient to take an additional Type-II right censored data from another independent sample (possibly of small size $s$ ), it might be possible to use the combined ordered sample from these two Type-II right censored samples in order to increase the precision of the estimation. There are a variety of scenarios wherein one can obtain combined ordered sample from two independent Type-II censored samples arising from a common parent distribution. One possible situation is when the number of items placed on a life test per run is limited, several independent runs need to be done. Another scenario is in the context of a meta-analysis when similar life-testing experiments from different facilities need to be pooled together.

From [1], the situation in which two independent Type-II right censored samples are pooled, and the advantage of pooling samples is demonstrated and the joint distribution of order statistics from the pooled sample as a mixture of progressively Type-II censored samples is expressed. Using these mixture forms, the nonparametric prediction intervals are then derived for order statistics from a future sample. In this paper, we discuss the problem of estimating the unknown parameters of the left truncated exponential distribution and predicting the failure times from a future sample from the sample population when the observed sample is a pooled sample from two independent Type-II right censored samples.

For the Bayesian estimation in this context, we consider here three types of loss functions. The first is the squared error (SE) loss function which is a symmetric function that gives equal importance to overestimation and 
underestimation in the parameter estimation. The second is the linear-exponential (LINEX) loss function, introduced by Varian in [2], which is asymmetric and gives differing weights to overestimation and underestimation. This function rises approximately exponentially on one side of zero and approximately linearly on the other side. These loss functions have been used by many authors; see, for example, [3], [4], [5], [6], [7], [8], and [9]. The third loss function is the generalization of the entropy (GE) loss function, used by several authors (see, for example, [10]). This more general version allows for different shapes of the loss function.

In many practical problems, one may wish to use past data to predict an observation from a future sample from the same population. As in the case of estimation, a predictor can be either a point or an interval. Bayesian prediction for future observations from the exponential distribution has been discussed by many authors, including [11], [12], [13], [14], [15], [16], [17], [18], and [19].

The rest of this paper is organized as follows. In Section 2, the description of the model of the pooled sample from two independent Type-II censored samples is presented. The maximum likelihood (ML) estimator and the Bayesian estimators of the unknown parameters under SE, LINEX, and GE loss functions are derived in Section 3. The problem of predicting the order statistics from a future sample is discussed in Section 4. Finally, in Section 5, some computational results are presented for illustrating all the inferential methods developed here.

\section{The Model Description}

Let $X_{1: n}, \ldots, X_{r: n}$ and $Y_{1: m}, \ldots, Y_{s: m}$ be independent right Type-II censored samples from two independent random samples $X_{1}, \ldots, X_{n}$ and $Y_{1}, \ldots, Y_{m}$, respectively, drawn from a population with distribution function $F$. In the following, the pooled sample from $X_{1: n}, \ldots, X_{r: n} ; Y_{1: m}, \ldots, Y_{s: m}$ will be denoted by $Z=\left(Z_{(1)}, \ldots, Z_{(r+s)}\right)$ where $Z_{(1)} \leq \cdots \leq Z_{(r+s)}$.

The joint density function of $Z=\left(Z_{(1)}, \ldots, Z_{(r+s)}\right)$ is derived as a mixture of progressively Type-II censored samples given by

$$
f_{Z}(Z)=\sum_{i=0}^{r-1} \beta_{i} f_{T_{i}}(Z)+\sum_{j=0}^{s-1} \beta_{j}^{*} f_{T_{j}^{*}}(Z),
$$

where $Z=\left(Z_{(1)}, \ldots, Z_{(r+s)}\right)$ is a vector of realizations, $T_{i}=\left(T_{1: r+s: n+m}^{R_{i}}, \ldots, T_{r+s: r+s: n+m}^{R_{i}}\right)$ for $i=0, \ldots, r-1$, and $T_{j}^{*}=\left(T_{1: r+s: n+m}^{R_{j}^{*}}, \ldots, T_{r+s: r+s: n+m}^{R_{j}^{*}}\right)$ for $j=0, \ldots, s-1$, are progressively Type-II censored samples from the same population based on the progressive censoring schemes

$$
\begin{aligned}
& R_{i}=(\underbrace{0, \ldots, 0, m-s}_{s+i} \underbrace{0, \ldots, 0, n-r}_{r-i}), \\
& R_{j}^{*}=(\underbrace{0, \ldots, 0, n-r}_{r+j} \underbrace{0, \ldots, 0, m-s}_{s+j}),
\end{aligned}
$$

respectively, and the constants $\beta_{\mathrm{i}}$ and $\beta_{\mathrm{j}}^{*}$ are given by

$$
\begin{aligned}
& \beta_{i}=\frac{\left(\begin{array}{c}
s+i-1 \\
s-1
\end{array}\right)\left(\begin{array}{c}
m+n-s-i \\
n-i
\end{array}\right)}{\left(\begin{array}{c}
m+n \\
n
\end{array}\right)}, \text { for } i=0, \ldots, r-1, \\
& \beta_{j}^{*}=\frac{\left(\begin{array}{c}
r+j-1 \\
r-1
\end{array}\right)\left(\begin{array}{c}
m+n-r-j \\
m-j
\end{array}\right)}{\left(\begin{array}{c}
m+n \\
m
\end{array}\right)}, \text { for } j=0, \ldots, s-1 .
\end{aligned}
$$

By using the joint density function of the progressively Type-II censored sample, see [20] and [21], the joint density function in (2.1) becomes

$$
f_{Z}(Z)=\sum_{i=0}^{r-1} A_{i}\left[1-F\left(z_{s+i}\right)\right]^{m-s}\left[1-F\left(z_{r+s}\right)\right]^{n-r} \prod_{q=1}^{r+s} f\left(z_{q}\right)+\sum_{j=0}^{s-1} A_{j}^{*}\left[1-F\left(z_{r+j}\right)\right]^{n-r}\left[1-F\left(z_{r+s}\right)\right]^{m-s} \prod_{q=1}^{r+s} f\left(z_{q}\right)
$$

where

$$
\begin{gathered}
A_{i}=\beta_{i} K_{i}, K_{i}=\frac{(m+n) !(n-i) !}{(m+n-s-i) !(n-r) !}, \text { for } i=0, \ldots, r-1, \\
A_{j}^{*}=\beta_{j}^{*} K_{j}^{*}, K_{j}^{*}=\frac{(m+n) !(m-j) !}{(m+n-r-j) !(m-s) !}, \text { for } j=0, \ldots, s-1 .
\end{gathered}
$$

In this paper, the underlying distribution is assumed to be the left truncated exponential with probability density (PDF) and cumulative (CDF) functions as

$$
f(x \mid \theta, \mu)=\theta \exp [-\theta(x-\mu)]
$$

and

$$
F(x \mid \theta, \mu)=1-\exp [-\theta(x-\mu)]
$$

with rate parameter $\theta>0$, and location parameter $\mu>0$. If $\mu$ is not restricted to be nonnegative then (2.3) is more appropriately referred to as the two-parameter exponential distribution. Introducing distinctive names for these two distributions is necessary since it is only the former (with $\mu \geq 0$ ) which is really appropriate as a lifetime distribution model.

The reliability function $R(x)$ and the $p^{\text {th }}$ quantile $\xi_{p}$ of the left truncated exponential distribution are given, respectively, by

$$
R(x)=\exp [-\theta(x-\mu)], x \geq \mu, \theta>0,
$$

and

$$
\xi_{p}=\mu-\frac{\log (1-p)}{\theta}, 0 \leq p \leq 1
$$

\section{ML and Bayesian Estimation}

In this section, we derive the ML estimator and the Bayesian estimators under SE, LINEX and GE loss functions for the unknown parameters $\theta$ and $\mu$. Also, the ML estimator and the Bayesian estimators of the corresponding reliability and $p^{\text {th }}$ quantile functions are developed.

Using (2.2) and (2.3), the likelihood function of $\theta$ and $\mu$ 
based on the pooled sample $Z=\left(Z_{(1)}, \ldots, Z_{(r+s)}\right)$ can be written as

$$
L(\theta, \mu \mid z)=\sum_{i=0}^{r-1} A_{i} \theta^{r+s} \exp \left\{-\theta\left[u_{i}+(m+n)\left(z_{1}-\mu\right)\right]\right\}+\sum_{j=0}^{s-1} A_{j}^{*} \theta^{r+s} \exp \left\{-\theta\left[u_{j}^{*}+(m+n)\left(z_{1}-\mu\right)\right]\right\}
$$

where

$$
u_{i}=\sum_{q=1}^{r+s}\left(z_{q}-z_{1}\right)+\left(z_{s+i}-z_{1}\right)(m-s)+\left(z_{r+s}-z_{1}\right)(n-r) \text { for } i=1, \ldots, r-1
$$

and

$$
u_{j}^{*}=\sum_{q=1}^{r+s}\left(z_{q}-z_{1}\right)+\left(z_{r+s}-z_{1}\right)(m-s)+\left(z_{r+j}-z_{1}\right)(n-r) \text { for } j=1, \ldots, s-1 .
$$

\subsection{Estimation}

From (3.1), the log-likelihood function of $(\theta, \mu)$ is given by

$$
\log L(\theta, \mu \mid z)=\log \left\{\sum_{i=0}^{r-1} A_{i} \theta^{r+s} e^{-\theta\left[u_{i}+(m+n)\left(z_{1}-\mu\right)\right]}+\sum_{j=0}^{s-1} A_{j}^{*} \theta^{r+s} e^{-\theta\left[u_{j}^{*}+(m+n)\left(z_{1}-\mu\right)\right]}\right\}
$$

Now, the likelihood function is maximized with respect to $\mu$ by taking $\hat{\mu}_{M L}=z_{1}$. To maximize relative to $\theta$, differentiate (3.2) with respect to $\theta$ and solve the equation

$$
\frac{\partial \log L(\theta, \mu \mid z)}{\partial \theta}=0
$$

and so the ML estimator $\hat{\theta}_{M L}$ of $\theta$ is readily obtained by solving the following equation

$$
\sum_{i=0}^{r-1} A_{i}\left(r+s-\theta u_{i}\right) e^{-\theta u_{i}}+\sum_{j=0}^{s-1} A_{j}^{*}\left(r+s-\theta u_{j}^{*}\right) e^{-\theta u_{j}^{*}}=0 .
$$

By using the invariance property, the ML estimators of the reliability function and the $p^{\text {th }}$ quantile function can be obtained, respectively, as

$$
\hat{R}(x)_{M L}=\exp \left[-\hat{\theta}_{M L}\left(x-\hat{\mu}_{M L}\right)\right], x \geq \mu, \theta>0,
$$

and

$$
\begin{array}{r}
\widehat{\xi}_{p_{M L}}=\hat{\mu}_{M L}-\frac{\log (1-p)}{\widehat{\theta}_{M L}}, 0 \leq p \leq 1 . \quad \text { (3.5) } \begin{array}{l}
0, c>0, \text { see [13]. It follows the } \\
\text { density of }(\theta, \mu) \text { given } Z=z \text { i }
\end{array} \\
\pi^{*}(\theta, \mu)=I^{-1}\left\{\sum_{i=0}^{r-1} A_{i} \theta^{G} e^{-\theta\left[H_{i}+C(B-\mu)\right]}+\sum_{j=0}^{s-1} A_{j}^{*} \theta^{G} e^{-\theta\left[H_{j}^{*}+C(B-\mu)\right]}\right\}
\end{array}
$$

\subsection{Bayesian Estimation}

For Bayesian estimation, we use here the natural conjugate prior density function for $(\theta, \mu)$ given by

$$
\pi(\theta, \mu) \alpha \theta^{g} e^{-\{-\theta[h+c(b-\mu)]\}}, 0<\mu<b, \theta>0,
$$

where to be a proper density we must have $g>-1, h>$ $0, c>0$, see [13]. It follows that the corresponding posterior density of $(\theta, \mu)$ given $Z=z$ is given by

where

$$
g=r+s+g, C=c+m+n, B=\min \left(b, z_{1}\right), H_{i}=u_{i}+h+b c+(m+n) z_{1}-B C, H_{j}^{*}=u_{j}^{*}+h+b c+
$$
$(m+n) z_{1}-B C$, and $I$ is the normalizing constant given by

$$
\begin{gathered}
I=\int_{0}^{\infty} \int_{0}^{B}\left\{\sum_{i=0}^{r-1} A_{i} \theta^{G} e^{-\theta\left[H_{i}+C(B-\mu)\right]}+\sum_{j=0}^{s-1} A_{j}^{*} \theta^{G} e^{-\theta\left[H_{j}^{*}+C(B-\mu)\right]}\right\} d \theta d \mu=\frac{\Gamma(G)}{C}\left\{\sum_{i=0}^{r-1} A_{i}\left[\left(H_{i}\right)^{-G}-\left(H_{i}+C B\right)^{-G}\right]+\right. \\
\left.\sum_{j=0}^{s-1} A_{j}^{*}\left[\left(H_{j}^{*}\right)^{-G}-\left(H_{j}^{*}+C B\right)^{-G}\right]\right\},
\end{gathered}
$$

with $\Gamma($.$) denotes the complete gamma function.$

Hence, the Bayesian estimator of $\theta$ under the SE loss function is given by

$$
\hat{\theta}_{B S}=E[\theta]=\frac{I^{-1} \Gamma(G+1)}{C}\left\{\sum_{i=0}^{r-1} \frac{A_{i}}{\left[H_{i}\right]^{(G+1)}-\left[H_{i}+C B\right]^{(G+1)}}+\sum_{j=0}^{s-1} \frac{A_{j}^{*}}{\left[H_{j}^{*}\right]^{(G+1)}-\left[H_{j}^{*}+C B\right]^{(G+1)}}\right\}
$$

and the Bayesian estimator of $\mu$ under the SE loss function is given by 


$$
\hat{\mu}_{B S}=E[\mu]=\frac{I^{-1} \Gamma(G-1)}{C^{2}}\left\{\sum_{i=0}^{r-1} \frac{A_{i}}{B C(G-1)\left[H_{i}\right]^{(G)}+\left[H_{i}+C B\right]^{(G-1)}-\left[H_{i}\right]^{(G-1)}}+\sum_{j=0}^{S-1} \frac{A_{j}^{*}}{B C(G-1)\left[H_{j}^{*}\right]^{(G)}+\left[H_{j}^{*}+C B\right]^{(G-1)}-\left[H_{j}^{*}\right]^{(G-1)}}\right\} .
$$

The Bayesian estimator of $\theta$ under the LINEX loss function is given by

$$
\hat{\theta}_{B L}=\frac{-1}{v} \log \left\{E\left[e^{-v \theta}\right]\right\}=\frac{-1}{v} \log \left\{\frac{I^{-1} \Gamma(G)}{C}\left\{\sum_{i=0}^{r-1} \frac{A_{i}}{\left[H_{i}+v\right]^{(G)}-\left[H_{i}+v+C B\right]^{(G+1)}}+\sum_{j=0}^{s-1} \frac{A_{j}^{*}}{\left[H_{j}^{*}+v\right]^{(G)}-\left[H_{j}^{*}+v+C B\right]^{(G)}}\right\}\right\}
$$

and the Bayes estimator of $\mu$ under the LINEX loss function is given by

$$
\hat{\mu}_{B L}=\frac{-1}{v} \log \left\{E\left[e^{-v \mu}\right]\right\}=\frac{-1}{v} \log \left\{I^{-1} \Gamma(G+1) \int_{0}^{B} e^{-v \mu}\left\{\sum_{i=0}^{r-1} \frac{A_{i}}{\left[H_{i}+C(B-\mu)\right]^{(G+1)}}+\sum_{j=0}^{s-1} \frac{A_{j}^{*}}{\left[H_{j}^{*}+C(B-\mu)\right]^{(G+1)}}\right\} d \mu\right\} .
$$

The Bayesian estimator of $\theta$ under the GE loss function is given by

$$
\hat{\theta}_{B E}=\left[E\left(\theta^{-d}\right)\right]^{\frac{-1}{d}}=\left\{\frac{I^{-1} \Gamma(G-d)}{C}\left\{\sum_{i=0}^{r-1} \frac{A_{i}}{\left[H_{i}\right]^{(G-d)}-\left[H_{i}+C B\right]^{(G-d)}}+\sum_{j=0}^{s-1} \frac{A_{j}^{*}}{\left[H_{j}^{*}\right]^{(G-d)}-\left[H_{j}^{*}+C B\right]^{(G-d)}}\right\}\right\}^{\frac{-1}{d}}
$$

and Bayesian estimator of $\mu$ under the GE loss function is given by

$$
\hat{\mu}_{B E}=\left[E\left(\mu^{-d}\right)\right]^{\frac{-1}{d}}=\left\{I^{-1} \Gamma(G+1) \int_{0}^{B} \mu^{-d}\left\{\sum_{i=0}^{r-1} \frac{A_{i}}{\left[H_{i}+C(B-\mu)\right]^{(G+1)}}+\sum_{j=0}^{s-1} \frac{A_{j}^{*}}{\left[H_{j}^{*}+C(B-\mu)\right]^{(G+1)}}\right\} d \mu\right\}^{\frac{-1}{d}} .
$$

The Bayesian estimator of the reliability function under the SE loss function is given by

$$
\hat{R}(x)_{B S}=E\left[e^{-\theta(x-\mu)}\right]=\frac{I^{-1} \Gamma(G)}{G(C+1)}\left\{\sum_{i=0}^{r-1} \frac{A_{i}}{\left[H_{i}+x-B\right]^{G}-\left[H_{i}+x+C B\right]^{G}}+\sum_{j=0}^{s-1} \frac{A_{j}^{*}}{\left.\left[H_{j}^{*}+x-B\right]\right]^{G}-\left[H_{j}^{*}+x+C B\right]^{G}}\right\}
$$

and the Bayesian estimator of the $p^{\text {th }}$ quantile function under the SE loss function is given by

$$
\hat{\xi}_{\mathrm{pBS}}=\mathrm{E}[\mu]-\log (1-\mathrm{p}) E\left[\frac{1}{\theta}\right]=\hat{\mu}_{B S}-\log (1-p) \frac{I^{-1} \Gamma(G+1)}{C}\left\{\sum_{i=0}^{r-1} \frac{A_{i}}{\left[H_{i}\right]^{(G+1)}-\left[H_{i}+C B\right]^{(G+1)}}+\sum_{j=0}^{S-1} \frac{A_{j}^{*}}{\left[H_{j}^{*}\right]^{(G+1)}-\left[H_{j}^{*}+C B\right]^{(G+1)}}\right\} .
$$

\section{Bayesian Prediction of Order Statistics from a Future Sample}

Let $W_{1: \rho}, W_{2: \rho}, \ldots, W_{\rho: \rho}$ be the order statistics from a future random sample of size $\rho$ from the same population. We discuss here the Bayesian prediction of $W_{q: \rho}$, for $q=1, \ldots, \rho$, based on the observed pooled ordered sample $Z=$
$\left(Z_{(1)}, Z_{(2)}, \ldots, Z_{(\mathrm{r}+\mathrm{s})}\right)$. We derive the Bayesian predictive distribution for $W_{q: \rho}$, and then find the Bayesian point predictor and prediction interval for $W_{q: \rho}$.

It is well known that the marginal density function of the $q^{\text {th }}$ order statistic from a sample of size $\rho$ from a continuous distribution with $\operatorname{CDF} F(x)$ and $\operatorname{PDF} f(x)$ is given by

$$
f_{W_{q: \rho}}(w \mid \theta, \mu)=\frac{\rho !}{(q-1) !(\rho-q) !}[F(w)]^{q-1}[1-F(w)]^{\rho-q} f(w), w \geq 0,
$$

for $1 \leq q \leq \rho$; see [22].

Upon substituting (2.3) and (2.4) in (4.1), the marginal density function of the $W_{\mathrm{q}: \rho}$ becomes

$$
f_{W_{q: \rho}}(w \mid \theta, \mu)=\sum_{h=0}^{q-1} C_{h}(q) \theta e^{-\theta \delta(w-\mu)}, w>\mu, \quad 1 \leq q \leq \rho,
$$

where $\delta=\rho-q+h+1$ and $C_{h}(q)=(-1)^{h} \frac{\rho !}{(q-h-1) !(\rho-q) ! h !}$ for $h=0, \ldots, q-1$.

By forming the product of (3.7) and (4.2), integrating out $(\theta, \mu)$ over the set $\left\{(\theta, \mu): \theta>0,0<\mu<\min \left(B, W_{q: \rho}\right)\right\}$ and introducing the proportionality constant, the Bayesian predictive density function of $W_{q: \rho}$, given $Z=z$, is then

$$
f_{W_{\mathrm{q}: \rho}}^{*}(w \mid z)=\left\{\begin{array}{lr}
f_{1}(w \mid z) & 0<w<B \\
f_{2}(w \mid z) & w>B
\end{array}\right.
$$

where 


$$
\begin{aligned}
f_{1}(w \mid z)= & \int_{0}^{\infty} \int_{0}^{w} \pi^{*}(\theta, \mu) f_{W_{q: \rho}}(w \mid \theta, \mu) d \theta d \mu=I^{-1} \Gamma(G+1)\left\{\sum _ { i = 0 } ^ { r - 1 } \sum _ { h = 0 } ^ { q - 1 } \frac { A _ { i } C _ { h } ( q ) } { ( C + \delta ) } \left\{\left[H_{i}+C(B-w)\right]^{-(G+1)}-\left[H_{i}+C B+\right.\right.\right. \\
& \left.\left.\delta w]^{-(G+1)}\right\}+\sum_{j=0}^{s-1} \sum_{h=0}^{q-1} \frac{A_{i} C_{h}(q)}{(C+\delta)}\left\{\left[H_{j}^{*}+C(B-w)\right]^{-(G+1)}-\left[H_{j}^{*}+C B+\delta w\right]^{-(G+1)}\right\}\right\}
\end{aligned}
$$

and

$$
\begin{gathered}
f_{2}(w \mid z)=\int_{0}^{\infty} \int_{0}^{B} \pi^{*}(\theta, \mu) f_{W_{q: \rho}}(w \mid \theta, \mu) d \theta d \mu=I^{-1} \Gamma(G+1)\left\{\sum _ { i = 0 } ^ { r - 1 } \sum _ { h = 0 } ^ { q - 1 } \frac { A _ { i } C _ { h } ( q ) } { ( C + \delta ) } \left\{\left[H_{i}+\delta(w-B)\right]^{-(G+1)}-\left[H_{i}+C B+\right.\right.\right. \\
\left.\left.\delta w]^{-(G+1)}\right\}+\sum_{j=0}^{s-1} \sum_{h=0}^{q-1} \frac{A_{i} C_{h}(q)}{(C+\delta)}\left\{\left[H_{j}^{*}+\delta(w-B)\right]^{-(G+1)}-\left[H_{j}^{*}+C B+\delta w\right]^{-(G+1)}\right\}\right\}
\end{gathered}
$$

From (4.3), we simply obtain the predictive survival function of $W_{q: \rho}$, given $Z=z$, as

$$
\bar{F}_{W_{\mathrm{q}: \rho}}^{*}(t \mid z)=\left\{\begin{array}{lr}
\bar{F}_{1}(w \mid z) & 0<t<B, \\
\bar{F}_{1}(w \mid z) & t>B,
\end{array}\right.
$$

where

$$
\bar{F}_{1}(w \mid z)=\int_{t}^{B} f_{1}(w \mid z) d w+\int_{B}^{\infty} f_{1}(w \mid z) d w
$$

with

$$
\left.\begin{array}{c}
\int_{t}^{B} f_{1}(w \mid z) d w=I^{-1} \Gamma(G)\left\{\sum_{i=0}^{r-1} \sum_{h=0}^{q-1} \frac{A_{i} C_{h}(q)}{(C+\delta)}\left\{\frac{\left[H_{i}\right]^{-G}-\left[H_{i}+C(B-t)\right]^{-G}}{C}-\frac{\left[H_{i}+B(\delta+C)\right]^{-G}-\left[H_{i}+C B+\delta t\right]^{-G}}{\delta}\right\}+\sum_{j=0}^{S-1} \sum_{h=0}^{q-1} \frac{A_{j}^{*} C_{h}(q)}{(C+\delta)}\left\{\frac{\left[H_{j}^{*}\right]^{-G}-\left[H_{j}^{*}+C(B-t)\right]^{-G}}{C}-\right.\right. \\
\left.\frac{\left[H_{j}^{*}+B(\delta+C)\right]^{-G}-\left[H_{j}^{*}+C B+\delta t\right]^{-G}}{\delta}\right\}
\end{array}\right\}
$$

$$
\begin{gathered}
\int_{B}^{\infty} f_{2}(w \mid z) d w=I^{-1} \Gamma(G)\left\{\sum_{i=0}^{r-1} \sum_{h=0}^{q-1} \frac{A_{i} C_{h}(q)}{\delta(C+\delta)}\left\{\left[H_{i}\right]^{-G}-\left[H_{i}+B(C+\delta)\right]^{-G}\right\}+\sum_{j=0}^{S-1} \sum_{h=0}^{q-1} \frac{A_{j}^{*} C_{h}(q)}{\delta(C+\delta)}\left\{\left[H_{j}^{*}\right]^{-G}-\right.\right. \\
\left.\left.\left[H_{j}^{*}+B(C+\delta)\right]^{-G}\right\}\right\}
\end{gathered}
$$

and

$$
\begin{aligned}
\bar{F}_{2}(w \mid z)=\int_{t}^{\infty} f_{2}(w \mid z) d w=I^{-1} \Gamma(G) & \left\{\sum_{i=0}^{r-1} \sum_{h=0}^{q-1} \frac{A_{i} C_{h}(q)}{\delta(C+\delta)}\left\{\left[H_{i}+\delta(t-B)\right]^{-G}-\left[H_{i}+C B+\delta t\right]^{-G}\right\}\right. \\
& \left.+\sum_{j=0}^{s-1} \sum_{h=0}^{q-1}\left\{\frac{A_{j}^{*} C_{h}(q)}{\delta(C+\delta)}\left\{\left[H_{j}^{*}+\delta(t-B)\right]^{-G}-\left[H_{j}^{*}+C B+\delta t\right]^{-G}\right\}\right\}\right\} .
\end{aligned}
$$

The Bayesian point predictor of $W_{q: \rho}$, under SE loss function is the mean of the predictive density, given by

$$
\bar{W}_{q: \rho}=\int_{0}^{B} w f_{1}(w \mid z) d w+\int_{B}^{\infty} w f_{2}(w \mid z) d w
$$

where

$$
\begin{aligned}
\int_{0}^{B} w f_{1}(w \mid z) d w & =I^{-1} \Gamma(G)\left\{\sum_{i=0}^{r-1} \sum_{h=0}^{q-1} \frac{A_{i} C_{h}(q)}{(C+\delta)}\left\{\frac{B\left[H_{i}\right]^{-G}}{C}-\frac{\left[H_{i}\right]^{1-G}-\left[H_{i}+C B\right]^{1-G}}{(G-1) C^{2}}+\frac{B\left[H_{i}+B(\delta+C)\right]^{-G}}{\delta}+\frac{\left[H_{i}+B(\delta+C)\right]^{1-G}-\left[H_{i}+C B\right]^{1-G}}{(G-1) \delta^{2}}\right\}\right. \\
+ & \left.\sum_{j=0}^{S-1} \sum_{h=0}^{q-1} \frac{A_{j}^{*} C_{h}(q)}{(C+\delta)}\left\{\frac{B\left[H_{j}^{*}\right]^{-G}}{C}-\frac{\left[H_{j}^{*}\right]^{1-G}-\left[H_{j}^{*}+C B\right]^{1-G}}{(G-1) C^{2}}+\frac{B\left[H_{j}^{*}+B(\delta+C)\right]^{-G}}{\delta}+\frac{\left[H_{j}^{*}+B(\delta+C)\right]^{1-G}-\left[H_{j}^{*}+C B\right]^{1-G}}{(G-1) \delta^{2}}\right\}\right\}
\end{aligned}
$$

and

$$
\begin{aligned}
\int_{B}^{\infty} f_{2}(w \mid z) d w= & I^{-1} \Gamma(G)\left\{\sum_{i=0}^{r-1} \sum_{h=0}^{q-1} \frac{A_{i} C_{h}(q)}{(C+\delta)}\left\{\frac{B\left[H_{i}\right]^{-G}}{\delta}+\frac{\left[H_{i}\right]^{1-G}}{(G-1) \delta^{2}}-\frac{B\left[H_{i}+B(\delta+C)\right]^{-G}}{\delta}-\frac{\left[H_{i}+B(\delta+C)\right]^{1-G}}{(G-1) \delta^{2}}\right\}\right. \\
& \left.+\sum_{j=0}^{s-1} \sum_{h=0}^{q-1} \frac{A_{j}^{*} C_{h}(q)}{(C+\delta)}\left\{\frac{B\left[H_{j}^{*}\right]^{-G}}{\delta}+\frac{\left[H_{j}^{*}\right]^{1-G}}{(G-1) \delta^{2}}-\frac{B\left[H_{j}^{*}+B(\delta+C)\right]^{-G}}{\delta}-\frac{\left[H_{j}^{*}+B(\delta+C)\right]^{1-G}}{(G-1) \delta^{2}}\right\}\right\} .
\end{aligned}
$$


The Bayesian predictive bounds of a two-sided equi-tailed $100(1-\gamma) \%$ interval for $W_{q: \rho}, 1 \leq q \leq \rho$ can be obtained by solving the following two equations:

$$
\bar{F}_{W_{q: \rho}}^{*}(L \mid z)=1-\frac{\gamma}{2} \text { and } \bar{F}_{W_{q: \rho}}^{*}(U \mid z)=\frac{\gamma}{2}
$$

where $\bar{F}_{W_{q: \rho}}^{*}(t \mid z)$ is as in (4.6), $L$ and $U$ denote the lower and upper bounds, respectively.

For the highest posterior density (HPD) method, we need to solve the following two equations:

$$
\bar{F}_{W_{q: \rho}}^{*}\left(L_{W_{q: \rho}} \mid z\right)-\bar{F}_{W_{\mathrm{q}: \rho}}^{*}\left(U_{W_{q: \rho}} \mid z\right)=1-\gamma
$$

and

$$
f_{W_{q: \rho}}^{*}\left(L_{W_{q: \rho}} \mid z\right)-f_{W_{q: \rho}}^{*}\left(U_{\left.W_{q: \rho} \mid z\right)=0,}\right.
$$

where $f_{W_{q: \rho}}^{*}(w \mid z)$ is as in (4.3), $L_{W_{q: \rho}}$ and $U_{W_{q: \rho}}$ denote the HPD lower and upper bounds, respectively.

\section{Numerical Results and an Illustrative Example}

In this section, the ML and Bayesian estimates based on the SE, LINEX and GE loss functions are all compared by means of a Monte Carlo simulation study. A numerical example is finally presented to illustrate all the inferential results established in the preceding sections.

\subsection{Monte Carlo Simulation}

A simulation study is carried out for evaluating the performance of the ML estimate and all the Bayesian estimates and for examining the performance of the point and interval predictions discussed in the preceding sections. We chose the parameter $\theta$ to be $0.1,0.5$ and 1 , with $\mu=1$, and the two sample sizes as $(m, n)=(10,10)$ for different choices of $r$ and $s$, we computed the ML and Bayesian estimates of $\theta$ and $\mu$ under the SE, LINEX and GE loss functions using informative priors (IP) and Jeffreys' noninformative prior (NIP). We repeated this process 1000 times and computed the estimated risk (ER) for each estimate by using the root mean square error and also computed the bias risk (BR) for each case. The ER of all the estimates of $\theta$ and $\mu$, for $\theta=0.1,0.5$ and 1 , with $\mu=1$ is summarized in Tables 1,2 and 3, respectively. Also, the ER of all the estimates of $\theta$ and $\mu$ for $\mu=0.1$ and 0.5 , with $\theta=1$ is computed and they are summarized in Tables 4 and 5, respectively.

From Tables 1-3, we observe that, for the different choices of $\theta$, the estimated risks of the Bayesian estimates based on the LINEX, GE and SE loss functions are smaller than those of the ML estimates. We also observe that the estimated risks of all the estimates decrease with increasing $r$ and $s$ even when the sample sizes $m$ and $n$ are small. Moreover, a comparison of the results for the informative priors with the corresponding ones for Jeffreys' non-informative priors reveals that the former produces more precise results, as we would expect. Finally, we observe that the estimated risks of the ML estimates are close to the corresponding ones of the Bayesian estimates based on the SE loss function under Jeffreys' non-informative priors.

\subsection{Illustrative Example}

In order to illustrate all the inferential results established in the preceding sections, we consider the data given in [23] (Table 4.1, p. 462). The original data consists of 60 times to breakdown in minutes of an insulating fluid subjected to high-voltage stress. The data is partitioned by [23] into six groups, each with ten insulating fluids. These data have been analyzed by [24] by assuming two-parameter exponential distribution. We introduce here right censoring in the data from groups 1 (Group $X$ ) and 5 (Group $Y$ ) with $r=9$ and $s=8$, as shown in Table 4. Based on the data in Table 4, we computed the ML estimate and the Bayesian estimates of $\theta$ under the SE, LINEX (with $v=0.5$ ) and GE (with $c=0.5$ ) loss functions using informative prior with $(g, h, c, b)=$ $(0.1,1,1,0.5)$, and for a noninformative prior on $(\theta, \mu)$, $g \rightarrow-1, h \rightarrow 0, c \rightarrow 0$ and $b \rightarrow \infty$, so that $G=r+s-$ $1, C=m+n, B=z_{1}, H_{i}=u_{i}$ and $H_{j}^{*}=u_{j}^{*}$. Also, we computed the ML estimate and Bayesian estimates of the reliability (with $x=2$ ) and $p^{\text {th }}$ quantile (with $p=0.5$ ) functions. Moreover, we computed the point predictors as well as the bounds of the equi-tailed prediction intervals and the HPD intervals for future order statistics $W_{q: \rho}$ for $q=$ $1,2, \ldots, 10$ from a future sample of size $\rho=10$ from the same

\begin{tabular}{|c|c|c|c|c|c|c|c|c|c|c|c|}
\hline \multirow{2}{*}{$\boldsymbol{\theta}$} & \multirow{2}{*}{$r$} & \multirow{2}{*}{$s$} & & \multicolumn{2}{|c|}{$\widehat{\boldsymbol{\theta}}_{M L}$} & \multicolumn{2}{|c|}{$\widehat{\boldsymbol{\theta}}_{B S}$} & \multicolumn{2}{|c|}{$\widehat{\boldsymbol{\theta}}_{B L}$} & \multicolumn{2}{|c|}{$\widehat{\boldsymbol{\theta}}_{B E}$} \\
\hline & & & & EB & ER & EB & ER & EB & ER & EB & ER \\
\hline \multirow[t]{9}{*}{0.1} & 4 & 4 & IP & 0.0331 & 0.0688 & 0.0074 & 0.0358 & 0.0070 & 0.0354 & 0.0022 & 0.0318 \\
\hline & & & NIP & & & 0.0187 & 0.0556 & 0.0187 & 0.0548 & 0.0193 & 0.0468 \\
\hline & 6 & 4 & IP & 0.0264 & 0.0586 & 0.0067 & 0.0341 & 0.0064 & 0.0338 & 0.0014 & 0.0309 \\
\hline & & & NIP & & & 0.0153 & 0.0490 & 0.0153 & 0.0484 & 0.0158 & 0.0425 \\
\hline & 6 & 6 & IP & 0.0197 & 0.0465 & 0.0055 & 0.0307 & 0.0053 & 0.0305 & 0.0009 & 0.0283 \\
\hline & & & NIP & & & 0.0112 & 0.0399 & 0.0112 & 0.0396 & 0.0115 & 0.0358 \\
\hline & 8 & 6 & IP & 0.0169 & 0.0413 & 0.0049 & 0.0289 & 0.0047 & 0.0287 & 0.0008 & 0.0269 \\
\hline & & & NIP & & & 0.0097 & 0.0360 & 0.0097 & 0.0357 & 0.0099 & 0.0326 \\
\hline & 8 & 8 & IP & 0.0136 & 0.0355 & 0.0041 & 0.0263 & 0.0039 & 0.0262 & 0.0007 & 0.0248 \\
\hline
\end{tabular}
population.

Table 1. Values of the estimated risks of the ML and Bayes estimators for $\theta$ with different choices of $r$ and $s$. 


\begin{tabular}{|c|c|c|c|c|c|c|c|c|c|c|c|}
\hline \multirow{2}{*}{$\boldsymbol{\theta}$} & \multirow{2}{*}{$r$} & \multirow{2}{*}{$s$} & & \multicolumn{2}{|c|}{$\widehat{\boldsymbol{\theta}}_{M L}$} & \multicolumn{2}{|c|}{$\widehat{\boldsymbol{\theta}}_{B S}$} & \multicolumn{2}{|c|}{$\widehat{\boldsymbol{\theta}}_{B L}$} & \multicolumn{2}{|c|}{$\widehat{\boldsymbol{\theta}}_{B E}$} \\
\hline & & & & EB & ER & EB & ER & EB & ER & EB & ER \\
\hline \multirow{11}{*}{0.5} & & & NIP & & & 0.0076 & 0.0314 & 0.0076 & 0.0313 & 0.0078 & 0.0290 \\
\hline & 4 & 4 & IP & 0.1657 & 0.3440 & 0.0687 & 0.2198 & 0.0537 & 0.2080 & 0.0146 & 0.1895 \\
\hline & & & NIP & & & 0.0827 & 0.2760 & 0.0680 & 0.2576 & 0.0185 & 0.2345 \\
\hline & 6 & 4 & IP & 0.1322 & 0.2932 & 0.0593 & 0.2031 & 0.0501 & 0.1940 & 0.0145 & 0.1794 \\
\hline & & & NIP & & & 0.0677 & 0.2437 & 0.0564 & 0.2305 & 0.0162 & 0.2131 \\
\hline & 6 & 6 & IP & 0.0987 & 0.2324 & 0.0465 & 0.1759 & 0.0396 & 0.1697 & 0.0106 & 0.1593 \\
\hline & & & NIP & & & 0.0490 & 0.1989 & 0.0412 & 0.1911 & 0.0118 & 0.1795 \\
\hline & 8 & 6 & IP & 0.0845 & 0.2064 & 0.0410 & 0.1626 & 0.0350 & 0.1577 & 0.0086 & 0.1488 \\
\hline & & & NIP & & & 0.0421 & 0.1794 & 0.0355 & 0.1734 & 0.0103 & 0.1637 \\
\hline & 8 & 8 & IP & 0.0682 & 0.1774 & 0.0333 & 0.1454 & 0.0285 & 0.1417 & 0.0058 & 0.1351 \\
\hline & & & NIP & & & 0.0328 & 0.1569 & 0.0276 & 0.1527 & 0.0081 & 0.1458 \\
\hline \multirow[t]{10}{*}{1} & 4 & 4 & IP & 0.3314 & 0.6881 & 0.0506 & 0.3577 & 0.0134 & 0.3291 & 0.0353 & 0.3247 \\
\hline & & & NIP & & & 0.1644 & 0.5523 & 0.1078 & 0.4840 & 0.0491 & 0.4698 \\
\hline & 6 & 4 & IP & 0.2644 & 0.5864 & 0.0477 & 0.3418 & 0.0165 & 0.3183 & 0.0314 & 0.3142 \\
\hline & & & NIP & & & 0.1348 & 0.4877 & 0.0910 & 0.4381 & 0.0358 & 0.4267 \\
\hline & 6 & 6 & IP & 0.1975 & 0.4649 & 0.0399 & 0.3074 & 0.0157 & 0.2904 & 0.0207 & 0.2869 \\
\hline & & & NIP & & & 0.0976 & 0.3979 & 0.0670 & 0.3681 & 0.0260 & 0.3593 \\
\hline & 8 & 6 & IP & 0.1691 & 0.4128 & 0.0356 & 0.2894 & 0.0143 & 0.2754 & 0.0168 & 0.2721 \\
\hline & & & NIP & & & 0.0839 & 0.3590 & 0.0578 & 0.3361 & 0.0230 & 0.3277 \\
\hline & 8 & 8 & IP & 0.1364 & 0.3548 & 0.0294 & 0.2641 & 0.0121 & 0.2534 & 0.0114 & 0.2508 \\
\hline & & & NIP & & & 0.0654 & 0.3139 & 0.0451 & 0.2978 & 0.0190 & 0.2917 \\
\hline
\end{tabular}

Table 2. Values of the estimated risks of the ML and Bayes estimators for $\mu$ with different choices of $r$ and $s$.

\begin{tabular}{|c|c|c|c|c|c|c|c|c|c|c|c|}
\hline \multirow{2}{*}{$\boldsymbol{\theta}$} & \multirow{2}{*}{$r$} & \multirow{2}{*}{$s$} & & \multicolumn{2}{|c|}{$\widehat{\mu}_{M L}$} & \multicolumn{2}{|c|}{$\widehat{\mu}_{B S}$} & \multicolumn{2}{|c|}{$\widehat{\mu}_{B L}$} & \multicolumn{2}{|c|}{$\widehat{\mu}_{B E}$} \\
\hline & & & & EB & ER & EB & ER & EB & ER & EB & ER \\
\hline \multirow[t]{10}{*}{0.1} & \multirow[t]{2}{*}{4} & \multirow[t]{2}{*}{4} & IP & \multirow[t]{2}{*}{0.4656} & \multirow[t]{2}{*}{0.6270} & 0.0760 & 0.4086 & 0.0454 & 0.3965 & 0.1377 & 0.4481 \\
\hline & & & NIP & & & 0.1206 & 0.5253 & 0.0871 & 0.5050 & 0.0993 & 0.5343 \\
\hline & \multirow[t]{2}{*}{6} & \multirow[t]{2}{*}{4} & IP & \multirow[t]{2}{*}{0.4656} & \multirow[t]{2}{*}{0.6270} & 0.0703 & 0.4038 & 0.0392 & 0.3905 & 0.1465 & 0.4413 \\
\hline & & & NIP & & & 0.1193 & 0.5243 & 0.0860 & 0.5039 & 0.0989 & 0.5344 \\
\hline & \multirow[t]{2}{*}{6} & \multirow[t]{2}{*}{6} & IP & \multirow[t]{2}{*}{0.4656} & \multirow[t]{2}{*}{0.6270} & 0.0706 & 0.4013 & 0.0396 & 0.3884 & 0.1442 & 0.4378 \\
\hline & & & NIP & & & 0.1173 & 0.5264 & 0.0842 & 0.5073 & 0.0999 & 0.5391 \\
\hline & \multirow[t]{2}{*}{8} & \multirow[t]{2}{*}{6} & IP & \multirow[t]{2}{*}{0.4656} & \multirow[t]{2}{*}{0.6270} & 0.0723 & 0.4009 & 0.0415 & 0.3874 & 0.1401 & 0.4348 \\
\hline & & & NIP & & & 0.1166 & 0.5262 & 0.0836 & 0.5073 & 0.0999 & 0.5394 \\
\hline & \multirow[t]{2}{*}{8} & \multirow[t]{2}{*}{8} & IP & \multirow[t]{2}{*}{0.4656} & 0.6270 & 0.0694 & 0.3936 & 0.0383 & 0.3795 & 0.1440 & 0.4255 \\
\hline & & & NIP & & & 0.1156 & 0.5268 & 0.0827 & 0.5083 & 0.1003 & 0.5412 \\
\hline 0.5 & 4 & 4 & IP & 0.1039 & 0.1511 & 0.0024 & 0.1161 & 0.0063 & 0.1169 & 0.0253 & 0.1273 \\
\hline & & & NIP & & & 0.0112 & 0.1181 & 0.0159 & 0.1195 & 0.0411 & 0.1363 \\
\hline & 6 & 4 & IP & 0.1039 & 0.1511 & 0.0009 & 0.1153 & 0.0046 & 0.1160 & 0.0213 & 0.1247 \\
\hline & & & NIP & & & 0.0088 & 0.1168 & 0.0132 & 0.1179 & 0.0348 & 0.1317 \\
\hline & 6 & 6 & IP & 0.1039 & 0.1511 & 0.0011 & 0.1144 & 0.0023 & 0.1149 & 0.0165 & 0.1213 \\
\hline & & & NIP & & & 0.0061 & 0.1153 & 0.0100 & 0.1161 & 0.0279 & 0.1260 \\
\hline & 8 & 6 & IP & 0.1003 & 0.1431 & 0.0017 & 0.1057 & 0.0025 & 0.1125 & 0.0179 & 0.1120 \\
\hline & & & NIP & & & 0.0051 & 0.1150 & 0.0089 & 0.1157 & 0.0255 & 0.1245 \\
\hline & 8 & 8 & IP & 0.1014 & 0.1442 & 0.0006 & 0.1060 & 0.0025 & 0.1064 & 0.0143 & 0.1113 \\
\hline & & & NIP & & & 0.0036 & 0.1141 & 0.0071 & 0.1147 & 0.0217 & 0.1216 \\
\hline 1 & 4 & 4 & IP & 0.0520 & 0.0755 & 0.0056 & 0.0588 & 0.0068 & 0.0592 & 0.0110 & 0.0614 \\
\hline & & & NIP & & & 0.0073 & 0.0603 & 0.0087 & 0.0609 & 0.0140 & 0.0644 \\
\hline & 6 & 4 & IP & 0.0520 & 0.0755 & 0.0040 & 0.0582 & 0.0051 & 0.0585 & 0.0085 & 0.0601 \\
\hline & & & NIP & & & 0.0056 & 0.0592 & 0.0068 & 0.0597 & 0.0110 & 0.0621 \\
\hline & 6 & 6 & IP & 0.0520 & 0.0755 & 0.0021 & 0.0575 & 0.0031 & 0.0577 & 0.0058 & 0.0587 \\
\hline & & & NIP & & & 0.0037 & 0.0581 & 0.0048 & 0.0584 & 0.0080 & 0.0598 \\
\hline & 8 & 6 & IP & 0.0531 & 0.0751 & 0.0002 & 0.0557 & 0.0011 & 0.0559 & 0.0035 & 0.0567 \\
\hline & & & NIP & & & 0.0031 & 0.0578 & 0.0041 & 0.0581 & 0.0070 & 0.0593 \\
\hline & 8 & 8 & IP & 0.0531 & 0.0751 & 0.0002 & 0.0557 & 0.0011 & 0.0559 & 0.0035 & 0.0567 \\
\hline & & & NIP & & & 0.0022 & 0.0573 & 0.0031 & 0.0575 & 0.0056 & 0.0583 \\
\hline
\end{tabular}


Table 3. The estimated bias and risk of the ML and Bayes estimators for the reliability function (with $x=2$ ) and $p^{\text {th }}$ quantile function (with $p=0.5$ ) for different choices of $r$ and $s$.

\begin{tabular}{|c|c|c|c|c|c|c|c|c|c|c|c|}
\hline \multirow{2}{*}{$\boldsymbol{\theta}$} & \multirow{2}{*}{$r$} & \multirow{2}{*}{$s$} & & \multicolumn{2}{|c|}{$\widehat{\boldsymbol{R}}_{M L}$} & \multicolumn{2}{|c|}{$\widehat{\boldsymbol{R}}_{B S}$} & \multicolumn{2}{|c|}{$\hat{\boldsymbol{t}}_{M L}$} & \multicolumn{2}{|c|}{$\hat{\boldsymbol{t}}_{B S}$} \\
\hline & & & & EB & ER & EB & ER & EB & ER & EB & ER \\
\hline \multirow[t]{10}{*}{0.1} & \multirow[t]{2}{*}{4} & \multirow[t]{2}{*}{4} & IP & \multirow[t]{2}{*}{0.0381} & \multirow[t]{2}{*}{0.0972} & 0.0096 & 0.0724 & \multirow[t]{2}{*}{0.2698} & \multirow[t]{2}{*}{2.4694} & 0.8043 & 2.6038 \\
\hline & & & NIP & & & 0.0073 & 0.0751 & & & 1.0393 & 3.0730 \\
\hline & \multirow[t]{2}{*}{6} & \multirow[t]{2}{*}{4} & IP & \multirow[t]{2}{*}{0.0403} & \multirow[t]{2}{*}{0.0887} & 0.0099 & 0.0663 & \multirow[t]{2}{*}{0.1092} & \multirow[t]{2}{*}{2.3490} & 0.7133 & 2.4491 \\
\hline & & & NIP & & & 0.0081 & 0.0678 & & & 0.8890 & 2.8056 \\
\hline & \multirow[t]{2}{*}{6} & \multirow[t]{2}{*}{6} & IP & \multirow[t]{2}{*}{0.0433} & \multirow[t]{2}{*}{0.0868} & 0.0112 & 0.0653 & \multirow[t]{2}{*}{0.0072} & \multirow[t]{2}{*}{2.0760} & 0.5899 & 2.1470 \\
\hline & & & NIP & & & 0.0098 & 0.0656 & & & 0.7129 & 2.3859 \\
\hline & \multirow[t]{2}{*}{8} & 6 & IP & 0.0442 & 0.0851 & 0.0114 & 0.0641 & 0.0857 & 1.9997 & 0.5540 & 2.0528 \\
\hline & & & NIP & & & 0.0101 & 0.0641 & & & 0.6568 & 2.2535 \\
\hline & 8 & 8 & IP & 0.0456 & 0.0835 & 0.0120 & 0.0625 & 0.1479 & 1.8030 & 0.4823 & 1.8378 \\
\hline & & & NIP & & & 0.0109 & 0.0621 & & & 0.5604 & 1.9840 \\
\hline 0.5 & 4 & 4 & IP & 0.0362 & 0.1380 & 0.0126 & 0.0998 & 0.0540 & 0.4939 & 0.1855 & 0.5431 \\
\hline & & & NIP & & & 0.0146 & 0.1152 & & & 0.2447 & 0.6499 \\
\hline & 6 & 4 & IP & 0.0235 & 0.1235 & 0.0108 & 0.0947 & 0.0218 & 0.4698 & 0.1576 & 0.5030 \\
\hline & & & NIP & & & 0.0121 & 0.1063 & & & 0.1994 & 0.5811 \\
\hline & 6 & 6 & IP & 0.0106 & 0.1048 & 0.0077 & 0.0853 & 0.0014 & 0.4152 & 0.1238 & 0.4340 \\
\hline & & & NIP & & & 0.0081 & 0.0927 & & & 0.1512 & 0.4843 \\
\hline & 8 & 6 & IP & 0.0048 & 0.0984 & 0.0063 & 0.0819 & 0.0171 & 0.3999 & 0.1131 & 0.4125 \\
\hline & & & NIP & & & 0.0066 & 0.0880 & & & 0.1352 & 0.4539 \\
\hline & 8 & 8 & IP & 0.0021 & 0.0881 & 0.0043 & 0.0752 & 0.0296 & 0.3606 & 0.0945 & 0.3662 \\
\hline & & & NIP & & & 0.0044 & 0.0795 & & & 0.1104 & 0.3956 \\
\hline 1 & 4 & 4 & IP & 0.0464 & 0.1430 & 0.0236 & 0.1062 & 0.0270 & 0.2469 & 0.1407 & 0.2925 \\
\hline & & & NIP & & & 0.0055 & 0.1245 & & & 0.1241 & 0.3276 \\
\hline & 6 & 4 & IP & 0.0349 & 0.1314 & 0.0200 & 0.1020 & 0.0109 & 0.2349 & 0.1179 & 0.2670 \\
\hline & & & NIP & & & 0.0045 & 0.1165 & & & 0.1004 & 0.2917 \\
\hline & 6 & 6 & IP & 0.0240 & 0.1143 & 0.0161 & 0.0929 & 0.0007 & 0.2076 & 0.0919 & 0.2275 \\
\hline & & & NIP & & & 0.0040 & 0.1032 & & & 0.0758 & 0.2425 \\
\hline & 8 & 6 & IP & 0.0183 & 0.1083 & 0.0149 & 0.0898 & 0.0086 & 0.2000 & 0.0831 & 0.2151 \\
\hline & & & NIP & & & 0.0040 & 0.0986 & & & 0.0677 & 0.2271 \\
\hline & 8 & 8 & IP & 0.0120 & 0.0969 & 0.0129 & 0.0826 & 0.0148 & 0.1803 & 0.0689 & 0.1899 \\
\hline & & & NIP & & & 0.0040 & 0.0892 & & & 0.0552 & 0.1977 \\
\hline
\end{tabular}

Table 4. Times to breakdown of insulating fluids from [23].

\begin{tabular}{|c|c|c|c|c|c|c|c|c|c|c|}
\hline Group X & 0.31 & 0.66 & 1.54 & 1.70 & 1.82 & 1.89 & 2.17 & 2.24 & 4.03 & * \\
\hline Group Y & 0.20 & 0.78 & 0.80 & 1.08 & 1.13 & 2.44 & 3.17 & 5.55 & * & * \\
\hline
\end{tabular}

Table 5. The $M L$ and Bayes estimates for $\theta, \mu, R(2)$ and $\xi_{0.5}$

\begin{tabular}{|c|c|c|c|c|c|c|c|c|c|c|c|c|}
\hline & $\widehat{\boldsymbol{\theta}}_{M L}$ & $\widehat{\boldsymbol{\theta}}_{B S}$ & $\widehat{\boldsymbol{\theta}}_{B L}$ & $\widehat{\boldsymbol{\theta}}_{B E}$ & $\widehat{\mu}_{M L}$ & $\widehat{\mu}_{B S}$ & $\widehat{\mu}_{B L}$ & $\widehat{\mu}_{B E}$ & $\widehat{R}_{M L}(2)$ & $\widehat{R}_{B S}(2)$ & $\widehat{\xi}_{0.5 M L}$ & $\widehat{\xi}_{0.5 B S}$ \\
\hline JP & 0.4290 & 0.4275 & 0.4249 & 0.4090 & 0.2000 & 0.1282 & 0.1275 & 0.0858 & 0.4620 & 0.4576 & 1.8159 & 1.8489 \\
\hline JP & & 0.4141 & 0.4115 & 0.3951 & & 0.1262 & 0.1255 & 0.0828 & & 0.4688 & & 1.9093 \\
\hline
\end{tabular}

Table 6. Bayesian prediction of $W_{q: \rho}$ for $q=1,2, \ldots, 10$.

\begin{tabular}{lcccccc}
\hline & \multicolumn{2}{c}{ Point predictor } & \multicolumn{2}{c}{ Equi-tailed interval } & \multicolumn{2}{c}{ HPD interval } \\
\hline $\mathbf{q}$ & IP & NIP & IP & NIP & IP & NIP \\
\hline 1 & 0.509 & 0.502 & $(0.208,1.220)$ & $(0.205,1.209)$ & $(0.200,1.012)$ & $(0.200,1.001)$ \\
2 & 0.652 & 0.669 & $(0.167,1.700)$ & $(0.167,1.763)$ & $(0.147,1.652)$ & $(0.145,1.703)$ \\
3 & 0.963 & 0.991 & $(0.275,2.333)$ & $(0.278,2.424)$ & $(0.119,1.990)$ & $(0.117,2.063)$ \\
4 & 1.317 & 1.358 & $(0.411,3.035)$ & $(0.418,3.157)$ & $(0.282,2.676)$ & $(0.283,2.778)$ \\
5 & 1.731 & 1.787 & $(0.581,3.847)$ & $(0.592,4.006)$ & $(0.416,3.418)$ & $(0.419,3.551)$ \\
6 & 2.227 & 2.302 & $(0.790,4.825))$ & $(0.806,5.030)$ & $(0.583,4.310)$ & $(0.590,4.482)$ \\
7 & 2.848 & 2.945 & $(1.051,6.067)$ & $(1.074,6.328)$ & $(0.792,5.437)$ & $(0.803,5.657)$ \\
8 & 3.676 & 3.802 & $(1.389,7.776)$ & $(1.421,8.114)$ & $(1.058,6.973)$ & $(1.074,7.258)$ \\
9 & 4.917 & 5.088 & $(1.860,10.495)$ & $(1.905,10.952)$ & $(1.413,9.378)$ & $(1.437,9.763)$ \\
10 & 7.399 & 7.661 & $(2.645,16.684)$ & $(2.711,17.401)$ & $(1.930,14.669)$ & $(1.965,15.268)$ \\
\hline
\end{tabular}




\section{Conclusions and Discussion}

In this paper, the ML estimation and the Bayesian estimation based on the SE, LINEX and GE loss functions for the unknown parameters of the left truncated exponential distributions have been discussed based on the pooled TypeII censored samples. Both Bayesian point and interval predictions of the future failures have been developed based on the observed pooled Type-II censored data. The ML and Bayesian estimates have then been compared through a Monte Carlo simulation study and a numerical example has also been presented to illustrate all the inferential results established here.

The computational results show that the Bayesian estimation based on the SE, LINEX and GE loss functions is more precise than the ML estimation. Also, the ERs of all the estimates decrease with increasing $r$ and $s$ even when the sample sizes $m$ and $n$ are small. Moreover, a comparison of the results for the informative priors with the corresponding ones for non-informative priors reveals that the former produce more precise results. Finally, the HPD prediction intervals seem to be more precise than the equi-tailed prediction intervals.

\section{References}

[1] Balakrishnan, N., Beutner, E., Cramer, E. (2010). Exact twosample non-parametric confidence, prediction, and tolerance intervals based on ordinary and progressively Type-II right censored data. Test, 19, 68-91.

[2] Varian, H. R. (1975). A Bayesian Approach to Real Estate Assessment. North-Holland, Amsterdam, The Netherlands.

[3] Rojo, J. (1987). On the admissibility of cX $+d$ with respect to the LINEX loss function. Communications in Statistics Theory and Methods, 16, 3745-3748.

[4] Basu, A. P., Ebrahimi, N. (1991). Bayesian approach to life testing and reliability estimation using asymmetric loss function. Journal of Statistical Planning and Inference, 29, 2131 .

[5] Pandey, B. N. (1997). Estimator of the scale parameter of the exponential distribution using LINEX loss function. Communications in Statistics - Theory and Methods, 26, 2191-2202.

[6] Soliman, A. A. (2002). Reliability estimation in a generalized life-model with application to the Burr-XII. IEEE Transactions on Reliability 51, 337-343.

[7] Soliman, A. A. (2005). Estimation of parameters of life from progressively censored data using Burr XII model. IEEE Transactions on Reliability, 54, 34-42.

[8] Soliman, A. A., Abd Ellah, A. H., Sultan, K. S. (2006). Comparison of estimates using record statistics from Weibull model: Bayesian and non-Bayesian approaches. Computational Statistics \& Data Analysis, 51, 2065-2077.
[9] Nassar, M. M., Eissa, F. H. (2004). Bayesian estimation for the exponentiated Weibull model. Communications in Statistics - Theory and Methods, 33, 2343-2362.

[10] Dey, D. K., Ghosh, M., Srinivasan, C. (1987). Simultaneous estimation of parameters under entropy loss. Journal of Statistical Planning and Inference, 15, 347-363.

[11] Dunsmore, I. R. (1974). The Bayesian predictive distribution in life testing models. Technometrics, 16, 455-460.

[12] Lingappaiah, G. S. (1979). Bayesian approach to prediction and the spacings in the exponential distribution. Annals of the Institute of Statistical Mathematics, 31, 391-401.

[13] Evans, I. G., Nigm, A. M. (1980). Bayesian prediction for the left truncated exponential distribution. Technometrics, 22, 201-204.

[14] AL-Hussaini, E. K., Jaheen, Z. F. (1999). Parametric prediction bounds for the future median of the exponential distribution. Statistics, 32, 267-275.

[15] Abdel-Aty, Y., Franz, J., Mahmoud, M. A. W. (2007). Bayesian prediction based on generalized order statistics using multiply type-II censoring. Statistics, 41, 495-504.

[16] Schenk, N., Burkschat, M., Cramer, E., Kamps, U. (2011). Bayesian estimation and prediction with multiply Type-II censored samples of sequential order statistics from one- and two-parameter exponential distributions. Journal of Statistical Planning and Inference, 141, 1575-1587.

[17] Mohie El-Din, M. M., Abdel-Aty, Y., Shafay, A. R. (2012). Two-sample Bayesian prediction intervals of generalized order statistics based on multiply Type II censored data. Communications in Statistics - Theory and Methods, 41, 381392.

[18] Mohie El-Din, M. M., Shafay, A. R. (2013). One- and twosample Bayesian prediction intervals based on progressively Type-II censored data. Statistical Papers, 54, 287-307.

[19] Shafay A. R., Balakrishnan, N., Sultan, K. s. (2014). Twosample Bayesian prediction for sequential order statistics from exponential distribution based on multiply Type-II censored samples. Journal of Statistical Computation and Simulation, $84,526-544$.

[20] Balakrishnan, N., Aggarwala, R. (2000). Progressive Censoring: Theory, Methods and Applications. Birkhauser, Boston.

[21] Balakrishnan, N. (2007). Progressive censoring methodology: An appraisal. Test 16, 211-296 (with discussions).

[22] Arnold, B.C., Balakrishnan, N., Nagaraja, H.N. (1992). A First Course in Order Statistics. John Wiley \& Sons, New York.

[23] Nelson, W. (1982). Applied Life Data Analysis. John Wiley \& Sons, New York.

[24] Balakrishnan, N., Lin, C. T., Chan, P. S. (2004). Exact inference and prediction for k-sample two-parameter exponential case under general Type-II censoring. Journal of Statistical Computation and Simulation, 74, 867-878. 